\section{Cureus}

\title{
The Bleeding Bowel: A Rare Case of Neurofibromatosis Type 1-associated Gastrointestinal Stromal Tumor in a Young Male
}

\author{
Ricci Kalayanamitra ${ }^{1}$, Zainab Shahid ${ }^{2}$, Neal Shah ${ }^{3}$, Ravi Patel $^{3}$, Rohit Jain ${ }^{3}$ \\ 1. Emergency Medicine, Penn State Health Milton S. Hershey Medical Center, Hershey, USA 2. Internal \\ Medicine, Lake Erie College of Osteopathic Medicine, Erie, USA 3. Internal Medicine, Penn State Health \\ Milton S. Hershey Medical Center, Hershey, USA
}

$\square$ Corresponding author: Ricci Kalayanamitra, ricci.kalaya@gmail.com Disclosures can be found in Additional Information at the end of the article

\section{Abstract}

Individuals with neurofibromatosis type 1 are much more likely to develop gastrointestinal stromal tumors than those without this condition. The median age for patients with neurofibromatosis type 1-associated gastrointestinal stromal tumors is approximately 65 years. We present a case of a young male with a history of neurofibromatosis type 1 who presented with symptomatic anemia and melena and was ultimately found to have a gastrointestinal stromal tumor.

Categories: Neurology, Gastroenterology, Oncology

Keywords: neurofibromatosis type 1, gastrointestinal stromal tumor, gist, nf1, nf1-associated gist, gi bleed, gastrointestinal bleed, melena, anemia, young

\section{Introduction}

Neurofibromatosis type 1 (NF1), also known as von Recklinghausen's disease, is the most common form of the three major clinically and genetically distinct classifications of neurofibromatosis (type 1, type 2 , and schwannnomatosis) [1]. It is an autosomal dominant condition that is caused by loss-of-function mutations in the tumor-suppressor gene NF1 and is characterized by cutaneous pigmentations and growth of tumors along nerves in the skin, brain, and other parts of the body [2]. According to the practice guideline for treating adults with NF1 by Medical Genetics and Genomics (ACMG) published in 2018, up to 1 in every 1,900 people is affected by NF1 worldwide. NF1 has been shown to decrease the average life expectancy of those affected by 8 to 15 years, most commonly due to malignancies and cardiovascular complications [3].

NF1 has a predilection for cancer development with a five-fold increase in overall cancer risk, which is the highest cancer incidence yet reported. It is closely associated with malignant peripheral nerve sheath tumors, breast cancers, and pheochromocytomas [4]. It has also been found to be associated with malignant fibrous histiocytomas, thyroid carcinomas and gastrointestinal stromal tumors (GISTs). The estimated cumulative cancer risk in patients with NF 1 was $25.1 \%$ by age 30 years and $38.8 \%$ by age 50 years, whereas the respective percentages in the general population of the same region were $0.8 \%$ and $3.9 \%$. The estimated lifetime cancer risk in patients with NF1 is $59.6 \%$, while it is approximately $38.5 \%$ in the general population [4-5].

\section{How to cite this article}

Kalayanamitra R, Shahid Z, Shah N, et al. (June 10, 2019) The Bleeding Bowel: A Rare Case of Neurofibromatosis Type 1-associated Gastrointestinal Stromal Tumor in a Young Male. Cureus 11(6): 
Patients with NF1 are 34 times more likely to develop GISTs than those without NF1 [6]. Although rare, GISTs are the most common mesenchymal tumors within the gastrointestinal tract [7]. A systematic review of 29 population-based studies involving 13,550 patients from 19 countries found that the reported incidence of GISTs overall was 10-15 people per million per year [8]. In contrast, the United States Cancer Statistics database from 2001 to 2015 revealed that the annual incidence of GISTs in the United States was seven cases per million people [9]. The primary locations of GISTs were $55.6 \%$ gastric, $31.8 \%$ small bowel, $6.0 \%$ colorectal, and $6.6 \%$ others. The ages of patients with GISTs ranged from 10 to 100 years, with the median age being roughly 65 years [8].

\section{Case Presentation}

A 21-year-old male with a past medical history of NF1 presented with shortness of breath, lethargy, and melena. He had been admitted to an outside hospital (OSH) two weeks prior with similar symptoms and was found to be anemic due to a gastrointestinal bleed. Colonoscopy and esophagogastroduodenoscopy (EGD) did not reveal any pathology, so the patient was discharged and scheduled for an outpatient capsule endoscopy. This was not performed due to his abrupt re-hospitalization to the OSH due to tachycardia and anemia with hemoglobin of 6 $\mathrm{g} / \mathrm{dL}$. Laboratory workup was non-contributory, so the patient was transfused with three units of packed red blood cells and was transferred to our institution [10].

The patient reported continued lethargy and denied abnormal bowel movements between his two earlier hospitalizations. His abdominal and rectal examinations were unremarkable, and skin examination revealed café-au-lait spots on the right arm. Hemoglobin was $8.5 \mathrm{mg} / \mathrm{dL}$, total bilirubin was $2.3 \mathrm{mg} / \mathrm{dL}$, and direct bilirubin was $0.3 \mathrm{mg} / \mathrm{dL}$. Vital signs were within normal limits. An abdominal computed tomography (CT) scan revealed a 2.45 x 2.04 x $3.51 \mathrm{~cm}$ wellcircumscribed ovoid mass at the jejunum adjacent to a loop of small bowel in the left upper quadrant (Figures $1 A, 1 B$ ). This mass was subsequently resected, biopsied, and confirmed as a low-grade GIST [10]. The surgical oncology team determined that he did not require any adjuvant chemotherapy or immunotherapy because his tumor was classified as low risk. The patient was ultimately discharged with pain control medications and was followed by his OSH care providers for any recurring mass. At one-year follow-up, he remained completely asymptomatic and free from cancer. 


\section{Cureus}
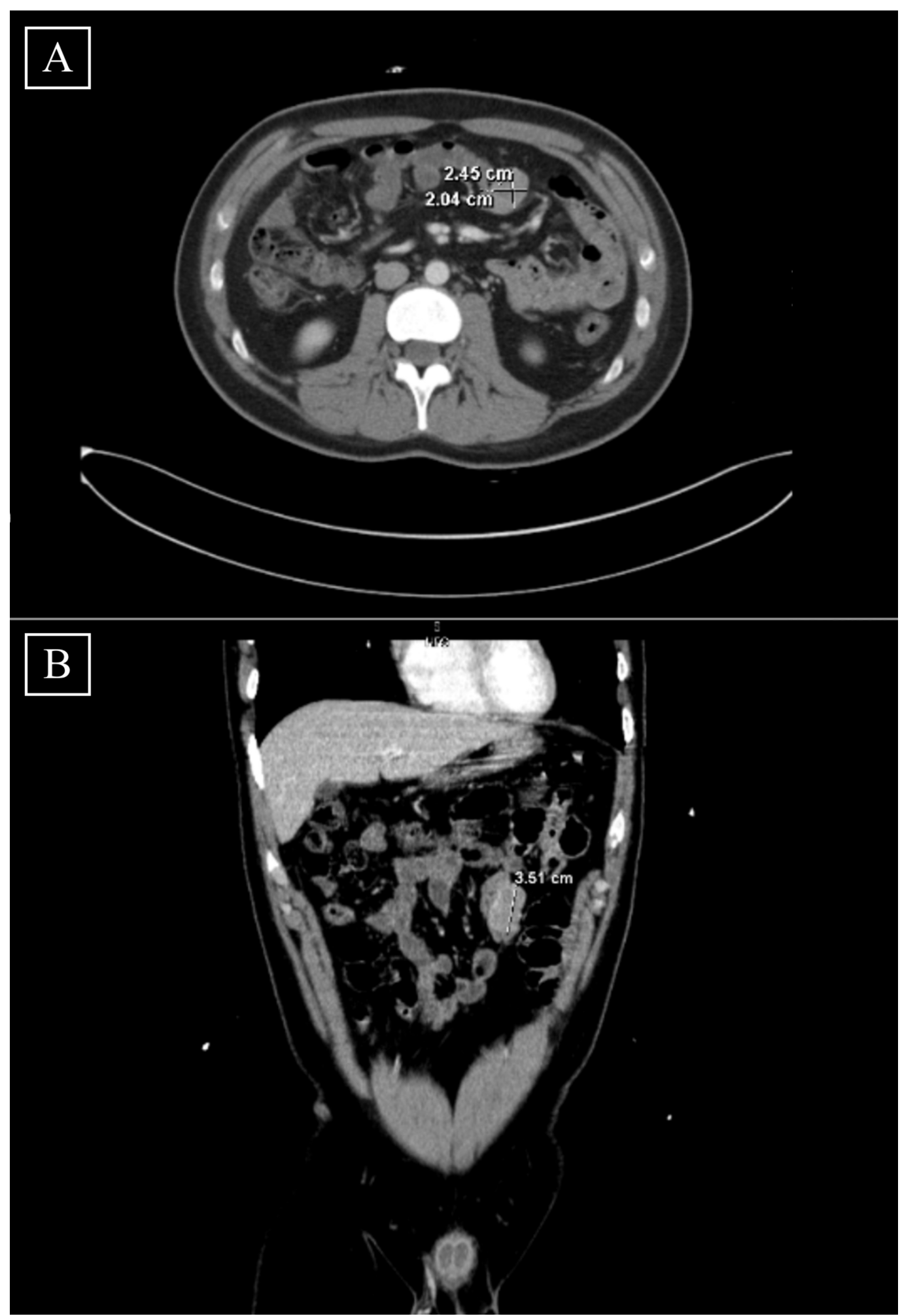

FIGURE 1: [A] Transverse view of CT scan revealing a $2.45 \mathrm{~cm}$ $x 2.04 \mathrm{~cm}$ well-circumscribed ovoid mass in the jejunum. [B] Coronal view of CT scan revealing a $3.51 \mathrm{~cm}$ wellcircumscribed ovoid mass in the jejunum.

CT: Computed tomography 


\section{Discussion}

The differential diagnoses of patients who present with anemia are broad. In the setting of anemia with melena, CT scans, EGDs, and colonoscopies are often ordered. If these examinations are non-revealing, patients are subjected to repeat studies or more specialized studies, such as capsule endoscopies and balloon enteroscopies. This adds to the physical and financial burdens of patients and may still fail to identify the underlying etiology of their symptoms. After admitting the patient in this case to our institution, we had initially planned for a push enteroscopy and a capsule study but were able to avoid this course by taking a thorough history of his NF1, researching its link to GISTs, and correlating this with the suspicious findings on imaging [10].

Without understanding this connection between NF1 and GISTs, achieving a prompt diagnosis would have been difficult given the patient's limited number of symptoms. Although our patient endorsed fatigue and had melena, he lacked abdominal pain, nausea, vomiting, bowel obstruction, a palpable abdominal mass, early satiety, dysphagia, and odynophagia, all of which have been reported as the most common signs and symptoms of GISTs [11]. His lack of symptoms is not unique, as a patient series conducted on 15 patients with NF1-associated GISTs found that GISTs were incidentally detected among seven patients while the other eight had symptomatic tumors [12]. This further accentuated the importance of linking NF1 and GISTs because it demonstrated that patients with NF1-associated GISTs could be asymptomatic.

Identifying this tumor early allows for prompt intervention and potential cure or halt of disease progression because GISTs are treatable tumors. Approximately $60 \%$ of GISTs are cured with surgery alone, especially in cases that involve low-to-moderate risk GISTs. With higher risk GISTs, a randomized phase III multi-center clinical trial demonstrated that patients who underwent surgery had significantly better recurrence-free survival and overall survival rates with three years of adjuvant imatinib therapy when compared to those who had only one year of adjuvant imatinib therapy [13]. Despite its rarity, providers should consider GISTs in patients with a history of NF1 who present with gastrointestinal symptoms, especially because there are effective treatment options for it.

This clinical suspicion should not be limited to older patients, despite the estimated median age for NF1-associated GISTs being 65 years, as the patient in this report was significantly younger [8]. A population-based prospective cohort study conducted on 1,404 NF1 patients from 1987 to 2012 reported 217 deaths throughout the study, with 107 (49\%) of them being due to cancer. The mortality rate was reported to be highest in young adulthood, between ages 15 and 30 years [4]. In addition, a previous case report highlighted the ability of NF1-associated GISTs to recur throughout a patient's life [14]. This, along with the mortality rate being higher in younger adults with NF1-associated GISTs, makes detecting the tumor in younger patients even more crucial. After having his GIST detected and removed at a young age, our patient can now be routinely monitored for any recurring mass throughout his life.

\section{Conclusions}

Providers should have a high index of suspicion for GISTs when taking care of patients with NF1 who present with gastrointestinal symptoms, regardless of age and/or a limited number of symptoms. Early detection of GISTs can minimize the financial and physical burdens associated with unnecessary diagnostic tests. Furthermore, since GISTs are treatable tumors, a prompt diagnosis can lead to effective treatment and prevention of disease progression.

\section{Additional Information}




\section{Disclosures}

Human subjects: Consent was obtained by all participants in this study. Conflicts of interest: In compliance with the ICMJE uniform disclosure form, all authors declare the following:

Payment/services info: All authors have declared that no financial support was received from any organization for the submitted work. Financial relationships: All authors have declared that they have no financial relationships at present or within the previous three years with any organizations that might have an interest in the submitted work. Other relationships: All authors have declared that there are no other relationships or activities that could appear to have influenced the submitted work.

\section{References}

1. Neurofibromatosis fact sheet. (2011). Accessed: May 13, 2019: https://www.ninds.nih.gov/Disorders/Patient-Caregiver-Education/FactSheets/Neurofibromatosis-Fact-Sheet.

2. Neurofibromatosis type 1. (2019). Accessed: May 15, 2019: https://ghr.nlm.nih.gov/condition/neurofibromatosis-type-1.

3. Stewart DR, Korf BR, Nathanson KL, Stevenson DA, Yohay K: Care of adults with neurofibromatosis type 1: a clinical practice resource of the American college of medical genetics and genomics (ACMG). Genet Med. 2018, 20:671-682. 10.1038/gim.2018.28

4. Uusitalo E, Rantanen M, Kallionpää RA, et al.: Distinctive cancer associations in patients with neurofibromatosis type 1. J Clin Oncol. 2016, 34:1978-1986. 10.1200/JCO.2015.65.3576

5. Sasieni PD, Shelton J, Ormiston-Smith N, Thomson CS, Silcocks PB: What is the lifetime risk of developing cancer?: the effect of adjusting for multiple primaries. Br J Cancer. 2011, 105:460-465. 10.1038/bjc.2011.250

6. Study links NF1 mutations to GIST tumors, highlighting the need for genetic testing . (2017). Accessed: May 13, 2019: https://www.oncology-central.com/diseasearea/gastrointestinal/study-links-nf1-mutations-gist-tumors-highlighting-nee....

7. Calabuig-Farinas S, Lopez-Guerrero JA, Llombart-Bosch A: The GIST paradigm: how to establish diagnostic and prognostic criteria. Arkh Patol. 2011, 73:13-21.

8. Søreide K, Sandvik OM, Søreide JA, Giljaca V, Jureckova A, Bulusu VR: Global epidemiology of gastrointestinal stromal tumours (GIST): a systematic review of population-based cohort studies. Cancer Epidemiol. 2016, 40:39-46. 10.1016/j.canep.2015.10.031

9. Patel N, Benipal B: Incidence of gastrointestinal stromal tumors in the United States from 2001-2015: a United States Cancer Statistics analysis of 50 states. Cureus. 2019, 11 :e4120. Accessed: June 8, 2019: 10.7759/cureus.4120

10. Kalayanamitra R, Luo JC, Shahid Z, Shah N, Patel R, Jain R: SGIM 2019 abstracts. J Gen Intern Med. 2019, 351. 10.1007/s11606-019-05007-5

11. Gastrointestinal stromal tumors: symptoms, causes, and risk factors . (2017). Accessed: May 9, 2019: https://www.healthline.com/health/gist/risk-factors-causes-and-symptoms\#1.

12. Andersson J, Sihto H, Meis-Kindblom JM, Joensuu H, Nupponen N, Kindblom LG: NF1associated gastrointestinal stromal tumors have unique clinical, phenotypic, and genotypic characteristics. Am J Surg Pathol. 2005, 29:1170-1176. 10.1097/01.pas.0000159775.77912.15

13. Joensuu H, Eriksson M, Hall KS, et al.: Adjuvant imatinib for high-risk GI stromal tumor: analysis of a randomized trial. J Clin Oncol. 2016, 34:244-250. 10.1200/JCO.2015.62.9170

14. Momani LAA, Abughanimeh O, Shipley LC, Phemister J, Swenson J, Young M: Recurrent gastric gastrointestinal stromal tumor in a patient with neurofibromatosis. Cureus. 2018, 10:e2854. Accessed: June 8, 2019: 10.7759/cureus.2854 